\title{
РАСПРЕДЕЛЕНИЕ НОВЫХ ПРОИЗВОДСТВЕННЫХ ПУНКТОВ НА РЫНКЕ И ПРОИЗВОДСТВЕННО-ТРАНСПОРТНЫЕ АСПЕКТЫ ПРЕДПРИЯТИЙ
}

В статье предложено комплексное решение трех задач линейного программирования: производственная задача (классическая постановка), транспортная задача, задача обслужсвания. Подобные задачи в предложсеной комплексной постановке часто возникают на предприятиях в прочессе расиирения производства в регионе или вхождения на новый рынок сбыта продукции. Рассмотрены основные алгоритмы поиска оптимального решения, сформулирована комплексная задача, построена модель и реализован алгоритм решения. Предложенная модель может быть использована на любом предприятии, которое открывает точки производства и при определении для всей организации оптимального объема выпуска. Целью таких организаций остается минимизация производственных издержек и затрат на транспортировку готовой продукции, а также получения максимальной прибьли.

Ключевые слова: комплексная задача, оптимизация, двудольный граф, логистика.

\section{Rodion Rogulin, Pavel Nechaev, Dmitry Pleshanov \\ DISTRIBUTION OF NEW INDUSTRIAL ITEMS ON THE MARKET AND INDUSTRIAL TRANSPORT ASPECTS OF ENTERPRISES}

This article proposes a comprehensive solution to the three linear programming problems: Production problem (classical formulation), Transport problem, Service problem. Similar tasks in the proposed complex formulation often arise at enterprises in the process of expanding production in the region or entering a new market for their products. The main algorithms for finding the optimal solution are considered, a complex problem is formulated, a model is built, and a solution algorithm is implemented. The proposed model can be used in any enterprise that opens production points and when determining the optimal output for the entire organization. The purpose of such organizations is to minimize production costs and the cost of transporting finished products, as well as maximizing profits.

Key words: complex task, optimization, bipartite graph, logistics.

Bвedeниe/Introduction. Любое предприятие в процессе хозяйственной деятельности стремится к минимизации издержек и максимизации прибыли при ограничениях на ресурсы. При всем многообразии методов оптимизации процессов управления ресурсами предприятий в научной литературе недостаточно представлены единые алгоритмы и модели для нахождения оптимального решения комплексных проблем хозяйственной деятельности предприятия. В каждой организации существуют нижеперечисленные главные задачи: задача производства (оптимальный выпуск продукции), транспортная задача (определение пути и объема перевозок по двудольным графам), задача максимального потока (нахождение максимального по объему перевозок пути на графе), задача минимизации времени, задача размещения центров сбыта (обслуживания, производства), задача распределения людских ресурсов при производстве. В нашей статье мы рассмотрим только три из них: задача производства (оптимальный выпуск продукции), транспортная задача, задача размещения центров производства - как единую комплексную задачу.

В общем виде оптимизационная задача - это экономико-математическая задача нахождения оптимального (максимального или минимального) значения целевой функции $f\left(x_{1}, \ldots, x_{n}\right)$ в некоторой допустимой области $W$ значений переменных $x_{i}, i=1,2, \ldots, n$. Математически в самом общем виде задача оптимизации записывается так:

$$
f\left(x_{1}, \ldots, x_{n}\right) \rightarrow \max ; x_{i} \in W_{i}=1,2, \ldots, n
$$


Maтериала и методы / Materials and methods. Для решения подобных задач используются алгоритмы определения оптимального решения (таблица 1)

Таблица 1

Сравнение основных алгоритмов поиска оптимального решения

\begin{tabular}{|l|c|c|}
\hline \multicolumn{1}{|c|}{ Название алгоритма } & Скорость сходимости & $\begin{array}{c}\text { Учитывает ли проблему } \\
\text { «Big Data» }\end{array}$ \\
\hline Метод Литтла - Гомори $[6,7]$ & Высокая & Нет \\
\hline Метод ветвей и границ $[8,9]$ & Низкая & Нет \\
\hline Генетический алгоритм $[10,11]$ & Низкая & Да \\
\hline
\end{tabular}

Метод Гомори представляет собой алгоритм отсечений путем генерации прямых (плоскостей, гиперплоскостей) и введением их в систему ограничений [6]. Метод ветвей и границ представляет собой дерево решений, конечным результатом которого является оптимальное решение $[8,12]$. Вышеперечисленные методы - достаточно быстрые алгоритмы для задач небольшой выборки. Однако эти алгоритмы не справляются с проблемой больших данных в отличие от разработанного сравнительно недавно генетического алгоритма [10]. Данный алгоритм и его модификации особенно хороши, когда мы говорим о задачах линейного программирования (ЛП) с большой выборкой. Главное в подобных задачах - это составить целевую функцию.

В нашей статье мы рассмотрим следующую комбинацию: задачу оптимального выпуска продукции, транспортную задачу и проблему определения оптимальных мест для открытия новых пунктов производства - все они являются задачами ЛП, что значительно упрощает нахождение оптимального решения. Для реализации алгоритма поиска оптимального решения воспользуемся алгоритмом Гомори, так как он имеет высокую скорость сходимости.

Сформулируем обобщенную постановку задачи: выбрать из указанных в списке пунктов для открытия нового производственного пункта, так чтобы суммарные издержки на открытие этого пункта и издержки по транспортировке из него до всех известных потребителей были минимальны, определить оптимальный объем выпуска продукции из соображений максимизации прибыли. Такая комплексная задача может быть полезна для предприятий с большим денежным и продуктовым оборотами, которые считают необходимым расшириться и открыть новые производственные пункты для дальнейшего освоения или завоевания рынка сбыта.

Последовательно сформулируем математическую постановку каждой из задач ЛП. Пусть $m$ - число конечных пунктов потребления, $n$ - число вершин в графе, определяющем маршрут перевозок; $m_{1}$ - типы производимого товара, $n_{1}$-типы сырья. Предположим, существует некоторый объем трудозатрат на производство каждого вида товара из исходного вида сырья. Обозначим сами нормы трудозатрат как

$$
A=\left\{A_{i j}\right\}, i=1: n_{1}, j=1: m_{1},
$$

где $A_{i j}$ - это элемент, соответствующий количеству ресурса $i$ для производства $j$ товара. Определим вектор цен реализации товара $j$, как

$$
P=\left\{p_{j}\right\}, j=1: m_{1}
$$

Пусть существует некоторое количество товара, определенное спросом потребителя (магазины, ИП и т. д.). Обозначим его

$$
a=\left\{a_{i}\right\}, i=1: m,
$$

Кроме того, определим затраты на перевозку товара из пункта $i$ в $j$, обозначим как

$$
C=\left\{c_{i_{1} j_{1}}\right\}, i_{1}=1: n, j_{1}=1: n
$$


Доопределим входные данные предельным объемом производства каждого пункта производства

$$
L_{j}, j=1: m_{1}
$$

Доопределим входные данные издержками на открытие предприятие в каждом рассмотренном пункте, как

$$
f=\left\{f_{j}\right\}, j=1: m_{1},
$$

Максимальное количество пунктов производства определим количеством $Q$. Для полноты набора данных остается определить количество запасов сырья, обозначим их как

$$
b=\left\{b_{i}\right\}, i=1: n_{1} \text {. }
$$

Транспортная задача. Пусть $x_{i_{1} j_{1}}$ есть количество товара, перевозимое из пункта $i_{1}$ в пункт $j_{1}[11,12]$.

Необходимо минимизировать расходы, тогда целевая функция примет вид $[1,13,14]$

$$
\sum_{i_{1}=1}^{n} \sum_{j_{1}=1}^{n} x_{i_{1} j_{1}} c_{i_{1} j_{1}} \rightarrow \min
$$

Учтем спрос потребителя, тогда $[15,16]$

$$
\sum_{i_{1}=1}^{n} x_{i_{1}, j}=a_{j}, \quad j=1: m
$$

Также, учтем запасы на складе, тогда

$$
\sum_{j_{1}=1}^{n} x_{i_{1}} \leq b_{i}, i=1: m
$$

Задача о размещении центров. Математическая модель подробно представлена в [1].

Производственная задача. Пусть $k_{j}$ есть количество товара $j$, которое подлежит производству из условия оптимума. Исходя из постановки задачи статьи, нужно максимизировать прибыль. Запишем это [1]

$$
\sum_{i=1}^{m_{1}} k_{j} p_{j} \rightarrow \max
$$

Исходя из соображений наличия запасов сырья, очевидно [1]

$$
\sum_{j=1}^{m_{1}} A_{i j} k_{j} \leq b_{i}, \quad i=1: n_{1} .
$$

Определим целочисленность продукции как [1]

$$
k_{j} \in Z^{+} \text {. }
$$

Мы предлагаем комплексное решение задачи $F_{0}$ оторое может быть записано следующим образом:

$$
\begin{gathered}
\sum_{i=1}^{m} \sum_{j=1}^{n} x_{i_{1} j_{1}} c_{i_{1} j_{1}}-\sum_{i=1}^{m_{1}}\left(k_{j} p_{j}+z_{j} f_{j}\right) \rightarrow \min , \\
\sum_{j=1}^{m_{1}} A_{i j} k_{j} \leq b_{i}, \quad i=1: n_{1},
\end{gathered}
$$




$$
\begin{gathered}
\sum_{i_{1}=1}^{m} x_{i_{1} j_{1}}=a_{j_{1}}, j_{1}=1: m, \\
\sum_{j_{1}=1}^{m} x_{i_{1} j_{1}}=k_{i_{1}}, i_{1}=1: m_{1}, \\
\sum z_{j} \leq Q, \\
k_{j} \leq L_{j} z_{j}, j=1: m_{1},
\end{gathered}
$$

где $z_{j}$ - открыт ли пункт $j$ для производства. $F_{0}$ ляется задачей линейного целочисленного программирования.

Подобные задачи часто возникают на любых производственных предприятиях: что производить и в каких объёмах, чтобы суммарная прибыль предприятия была максимальна с учетом минимизации издержек.

Задача $F_{0}$ может быть решена с помощью пакета Matlab. Ответ получим в виде одномерных массивов размерности $2 r+n^{2}$ Первые $r$ элементов отвечают за количество единиц произведенного товара. $n^{2}$ переменных - объем перевезенной продукции по каждой дуге. Остальные $r$ элементов отвечают за факт включения пункта производства в систему производства. Рассмотрим задачу $F_{0}$ подробнее.

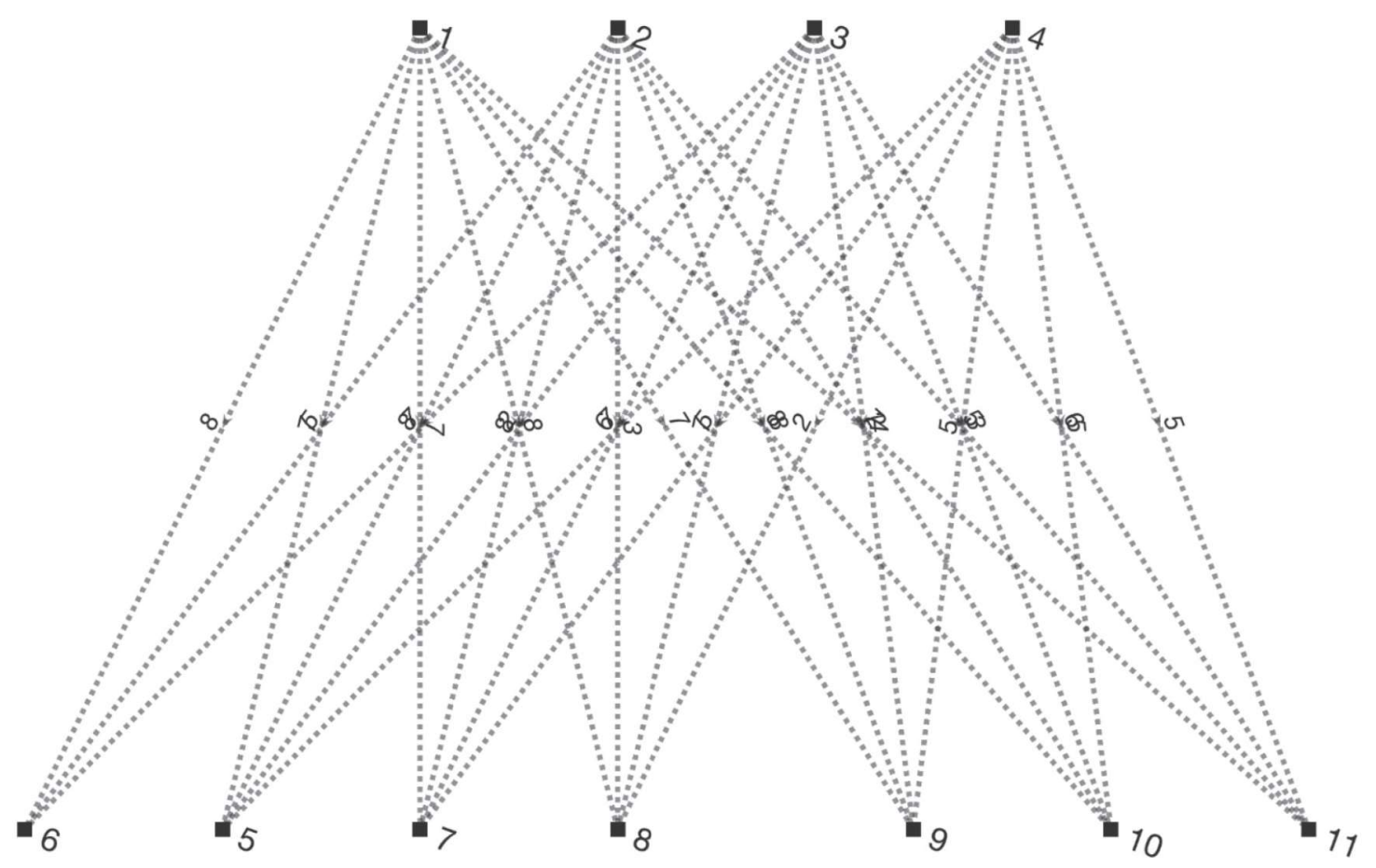

Рис. 1. Произвольная визуализация матрицы смежности $C$ - стоимости перевозок

Исходные данные: матрица стоимостей для перевозки с соответствующей вершины в следующую (матрица $C$ ), и матрица потребностей $a=(1,2,5,1,2,1,3)$ единиц в конечных пунктах, цены реализации $P=(4 ; 9 ; 10.5 ; 6.5)=(4,9,10.5,6.5)$ у. е., нормы затрат сырья на производство единицы каждого товара 


$$
A=\left(\begin{array}{llll}
1 & 2 & 6 & 2 \\
2 & 6 & 9 & 4 \\
5 & 7 & 1 & 5 \\
4 & 8 & 9 & 8
\end{array}\right)
$$

запасы ресурсов $\mathrm{b}=(40 ; 60 ; 70 ; 100)$ у. е. На рис. 1 можно увидеть произвольную визуализацию матрицы $C$. Номера вершин - пункты производства, промежуточные пункты и пункты потребления. Веса дуг матрицы $C$ - это издержки на перевозку каждой единицы продукции.

Решим задачу двумя способами: последовательно (код представлен в [17]) и комплексно (код представлен в [18]). Рассмотрим результаты решения (таблица 2). Количество пунктов производства $r=4$, первые четыре вершины графа

Результаты и обсуждение / Results and discussion. При одинаковом объеме производства (16 единиц продукции), суммарные затраты на перевозку при комплексном решении меньше и остатки сырья в пунктах производства меньше: 24 натур. ед. при последовательном и 18 натур. ед. при комплексном решении. Эффект от комплексного решения (прибыль) составляет 20 у. е. На рис. 2 и 3 проиллюстрированы логистические пути.

Таблиия 2

Сравнение результатов решения задачи $F_{0}$

\begin{tabular}{|l|c|c|}
\hline & Последовательно & Комплексно \\
\hline $\begin{array}{l}\text { Объем произведенной продукции в каждом } \\
\text { из } r \text { пунктов производства, шт. }\end{array}$ & $(12,0,4,0)$ & $(10,0,3,3)$ \\
\hline Суммарные затраты на перевозку, у. е. & 99 & 80 \\
\hline $\begin{array}{l}\text { Остатки сырья каждом из пунктов производства, } \\
\text { натур. ед. }\end{array}$ & $(4,0,6,16)$ & $(6,1,2,9)$ \\
\hline Эффект комплексного решения, у. е. & & 20 \\
\hline
\end{tabular}

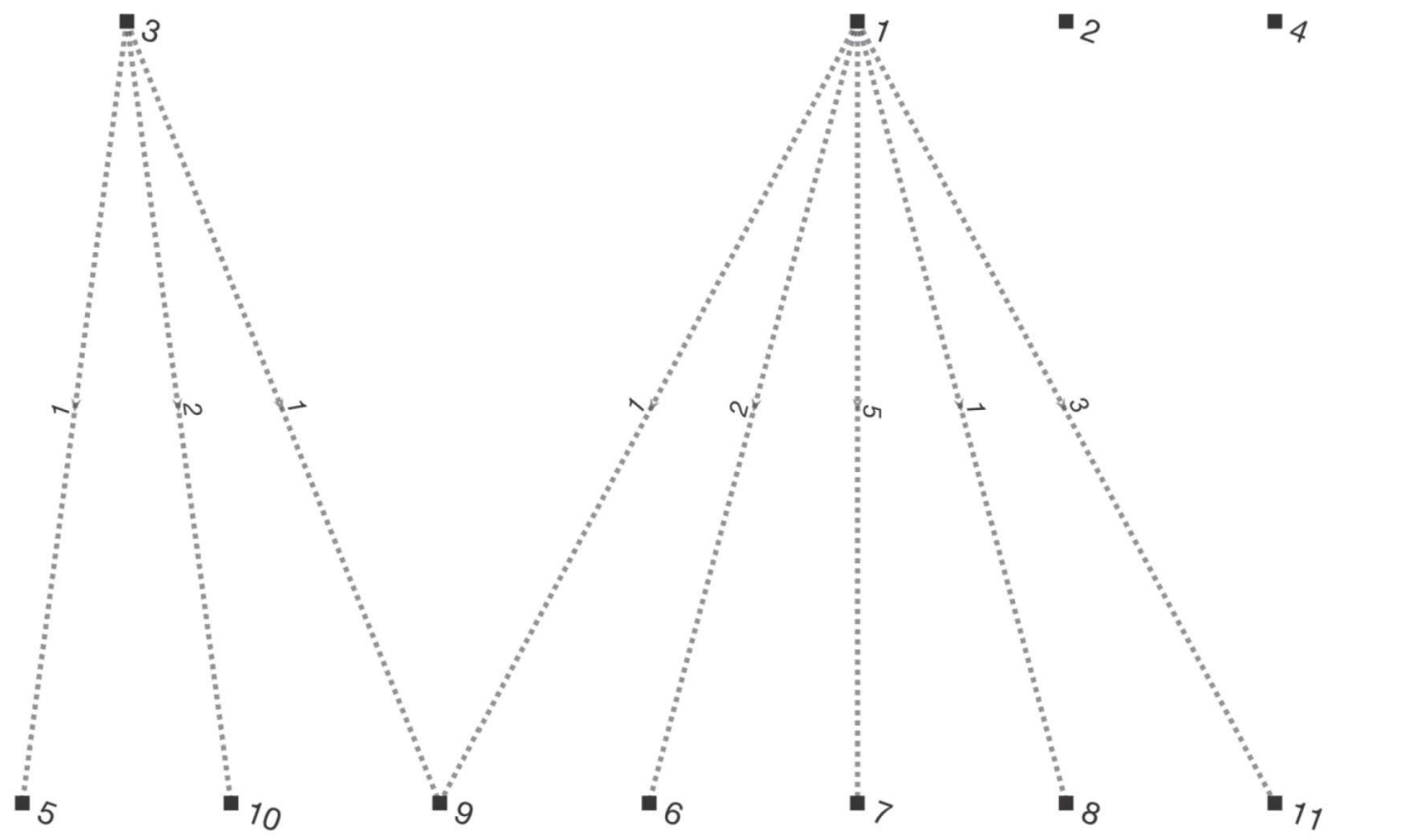

Рис. 2. Визуализация пути перевозок при решении задачи последовательно 


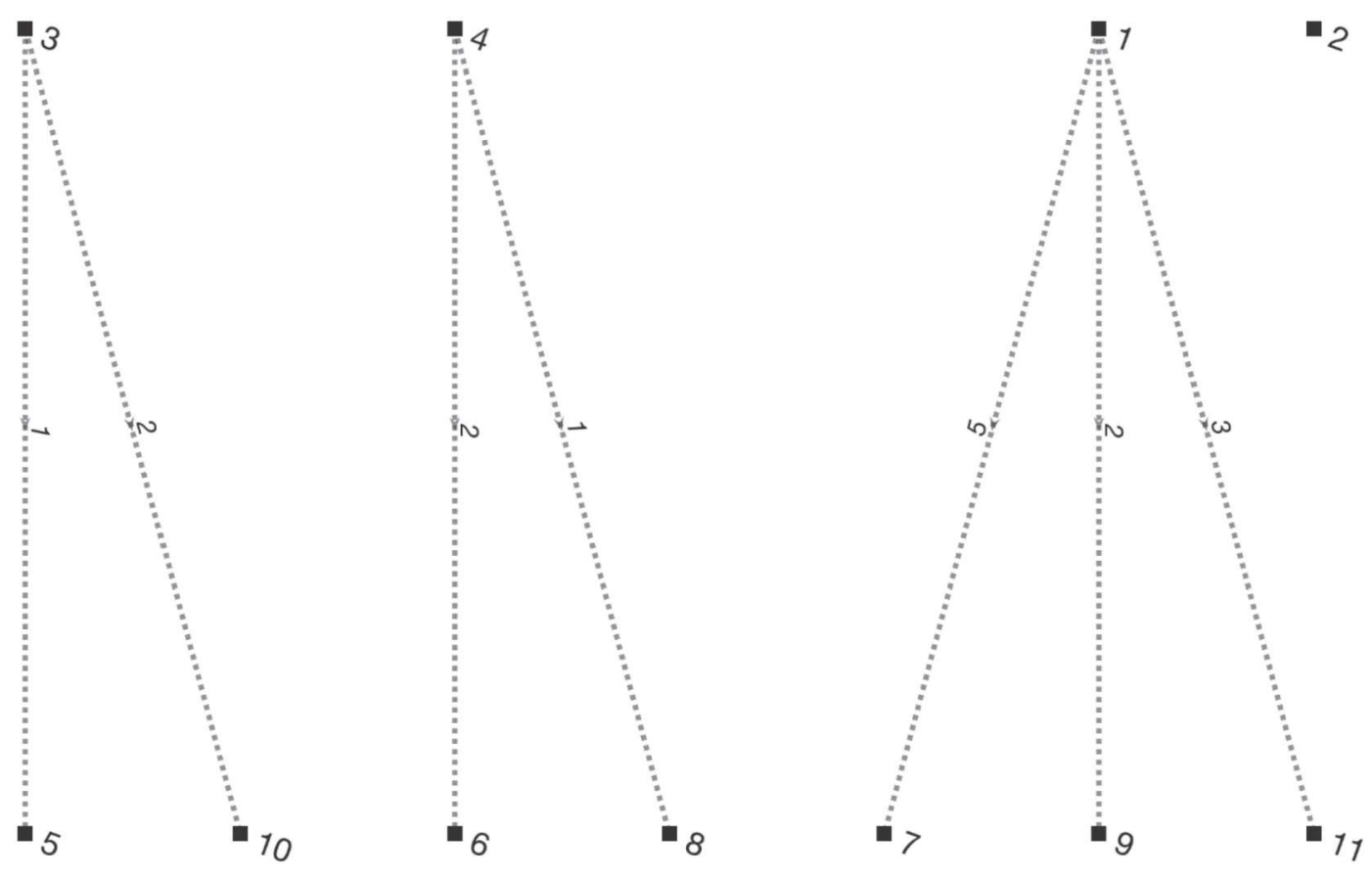

Рис. 3. Визуализация пути перевозок при решении задачи комплексно

Заключение / Conclusion. Предложенный подход обобщает ранее известные 3 классические задачи линейного программирования. Рассмотрены реальные данные предприятия при решении подобной задачи. Проведен анализ решения задачи. Комплексная постановка задачи и модель могут быть использованы на любом предприятии, где необходимо найти оптимальный комбинаторный вариант для производства с целью минимизации производственных издержек и затрат на транспортировку готовой продукции, а также получения максимальной прибыли. Такая модель является полезной при решении комплексных задач на предприятии.

\section{ЛИТЕРАТУРА И ИНТЕРНЕТ-РЕСУРСЫ}

1. Рогулин Р. С., Нечаев П. В., Плешанов Д. Е. Решение транспортной задачи линейного программирования с учетом времени и максимального потока // Транспортное дело России. 2018. № 4. С. 79-82.

2. Рогулин Р. С., Нечаев П. В., Плешанов Д. Е. Единая модель производственной, транспортной, учета времени, максимального потока // Экономика и предпринимательство. 2018. № 9 (98). С. 849-853.

3. Рогулин Р. С., Нечаев П. В., Плешанов Д. Е. Обобщение задач транспортной, учета времени, максимального потока в рамках единой экономической модели // Экономика и предпринимательство. 2018. № 9 (98). C. $813-816$.

4. Рогулин Р. С., Нечаев П. В., Плешанов Д. Е. Метод перекрестной энтропии для решения задачи коммивояжера // Современная наука: актуальные проблемы теории и практики. Серия: Естественные и технические науки. 2018. № 2. С. 52-58.

5. Pastebin. URL: https://pastebin.com/rxuOWNk2

6. Pastebin. URL: https://pastebin.com/Ecpnvfxm

\section{REFERENCES AND INTERNET RESOURCES}

1. Rogulin R. S., Nechaev P. V., Pleshanov D. E. Reshenie transportnoj zadachi linejnogo programmirovaniya $\mathrm{s}$ uchetom vremeni i maksimal'nogo potoka (Solution of the transport problem of linear programming taking into account time and maximum flow) // Transportnoe delo Rossii. 2018. № 4. S. 79-82. 
2. Rogulin R. S., Nechaev P. V., Pleshanov D. E. Edinaya model' proizvodstvennoj, transportnoj, ucheta vremeni, maksimal'nogo potoka (One model production, transport, accounting of time, maximum flow) // EHkonomika i predprinimatel'stvo. 2018. № 9 (98). S. 849-853.

3. Rogulin R. S., Nechaev P. V., Pleshanov D. E. Obobshchenie zadach transportnoj, ucheta vremeni, maksimal'nogo potoka $\mathrm{v}$ ramkah edinoj ehkonomicheskoj modeli (Generalization of the problems of transport, accounting of time, maximum flow in the from a united economic model) // EHkonomika i predprinimatel'stvo. 2018. № 9 (98). S. 813-816.

4. Rogulin R. S., Nechaev P. V., Pleshanov D. E. Metod perekrestnoj ehntropii dlya resheniya zadachi kommivoyazhera (The method of cross entropy for solving the traditional traffic problem) // Sovremennaya nauka: aktual'nye problemy teorii i praktiki. Seriya: Estestvennye i tekhnicheskie nauki. 2018. № 2. S. 52-58.

5. Pastebin. URL: https://pastebin.com/rxu0WNk2

6. Pastebin. URL: https://pastebin.com/Ecpnvfxm

\section{СВЕДЕНИЯ ОБ АВТОРАХ}

Рогулии Родион Сергеевич, магистрант второго курса кафедры информатики, математического и компьютерного моделирования, Дальневосточный Федеральный Университет. E-mail: rafassiaofusa@mail.ru

Нечаев Павел Владимирович, магистрант второго курса кафедры прикладной математики, механики, управления и программного обеспечения, Дальневосточный Федеральный Университет. E-mail: nechaev.pv@students.dvfu.ru

Плешанов Дмитрий Евгеньевич, магистрант второго курса кафедры прикладной математики, механики, управления и программного обеспечения, Дальневосточный Федеральный Университет. E-mail: pleshanov.de@students.dvfu.ru

\section{INFORMATION ABOUT AUTHORS}

Rodion Rogulin, Second-year master degree student of computer science, mathematical and computer modelling Department, Far Eastern Federal University, 89089935659, rafassiaofusa $@$ mail.ru

Pavel Nechaev, Second-year master degree student applied mathematics, mechanics, controlling and software Department, Far Eastern Federal University, nechaev.pv@students.dvfu.ru

Dmitry Pleshanov, Second-year master degree student applied mathematics, mechanics, controlling and software Department, Far Eastern Federal University, pleshanov.de@sstudents.dvfu.ru 